（62）埋設管異形管部の地震時しずん解析

川崎製鉄(株)，小池武

1. まえがき

埋設管路は直管・曲管・分岐管・人孔・バルブ室など多種類の要素棈造物が直列に連秸する形で一つの管路系 を楼成している。したが、て，最弱要素棈造物を見極の，“地震時安全性を模討することが，埋設管路システ

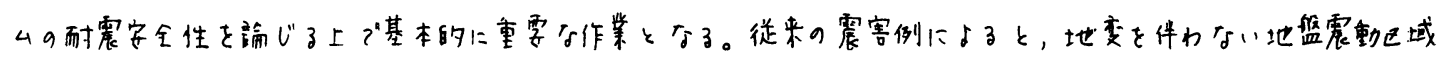

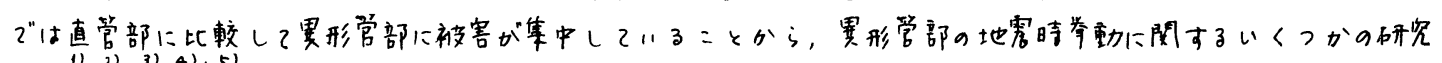

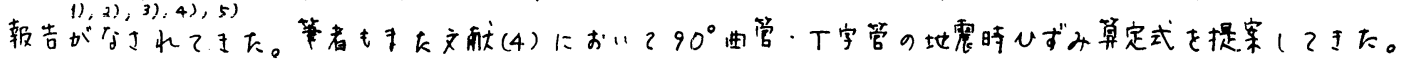

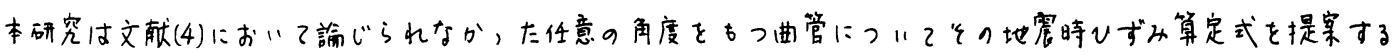

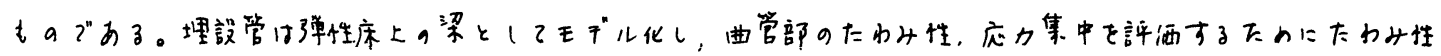
解析を实施(た。

\title{
2. 埋設管と周辺地盤間の相詨变位
}

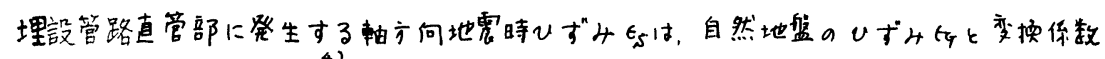
$\beta_{s}$ により次式で与之方孔る。

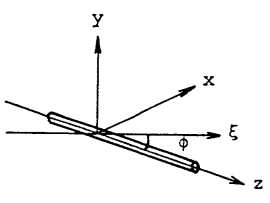

$$
\varepsilon_{S}=\beta_{S} \varepsilon_{G}
$$

Fig. 1

上式は，埋設管と周边地盤との間にすべリが発生しふい条件下での閐保式でろる。

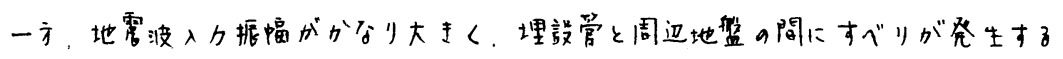

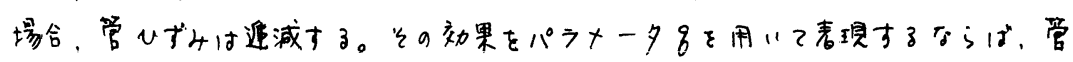

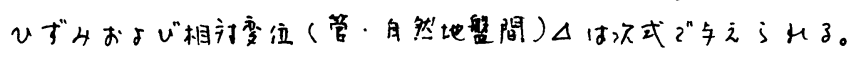

$$
\begin{aligned}
\varepsilon_{S} & =q_{S} \cdot \beta_{S} \cdot \varepsilon_{G} \\
\Delta & =\left(1-q_{S}^{*} \beta_{S}\right) v_{G}
\end{aligned}
$$

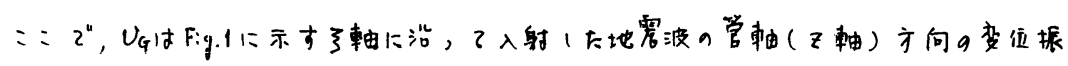

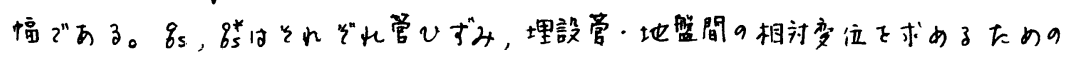

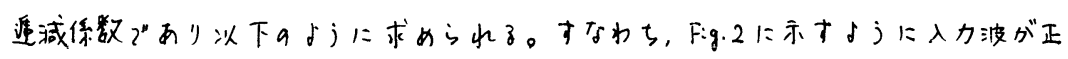

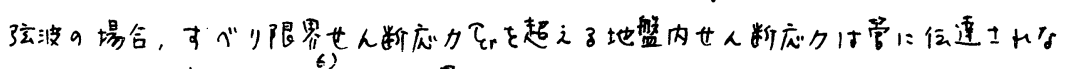
・とすると，宫内аオ法に徙，子最经的に次式飞得る。

$$
\begin{aligned}
& q_{S}=1-\frac{S\left(1+\kappa^{2}\right)-\sin n_{c r}}{k \sinh \left(k n_{c r}\right)} \\
& q_{S}^{*}=\left(1+\frac{1}{k^{2}}\right)\left\{\sin n_{c r}-S\left[1-\left\{\frac{\pi^{2}}{8}+\left(\frac{\eta_{c r}^{2}}{2}-\frac{\pi}{2} \eta_{c r}\right)\right\} k^{2}\right]\right\} \\
& =2^{*}, \quad \quad k=\lambda^{*} /\left(k_{\nu} \sin \phi\right), \lambda^{*}=\sqrt{K_{A} / E d \cdot 1 /\left\{1-\left(c_{a} / c_{A}\right)^{2}\right\}} \\
& S=\frac{1}{1+\kappa^{2}} \frac{\kappa \cos \eta_{c r} \tanh \left(\kappa \eta_{c r}\right)+\sin \eta_{c r}}{\kappa\left(\frac{\pi}{2}-\eta_{c r}\right) \tanh \left(\kappa \eta_{c r}\right)}
\end{aligned}
$$

Fig. 5

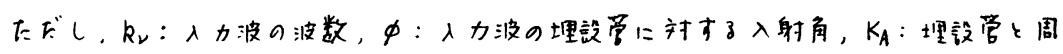

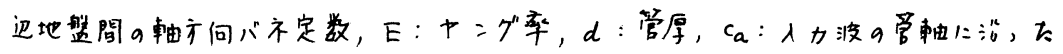

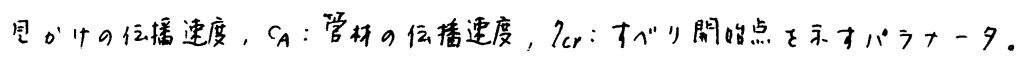

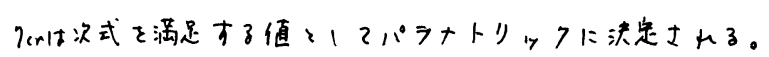

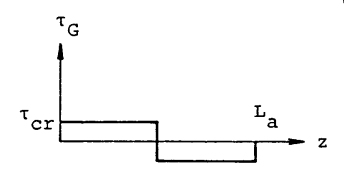

Fig. 3

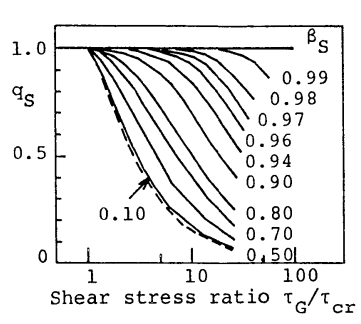

Fig. 4
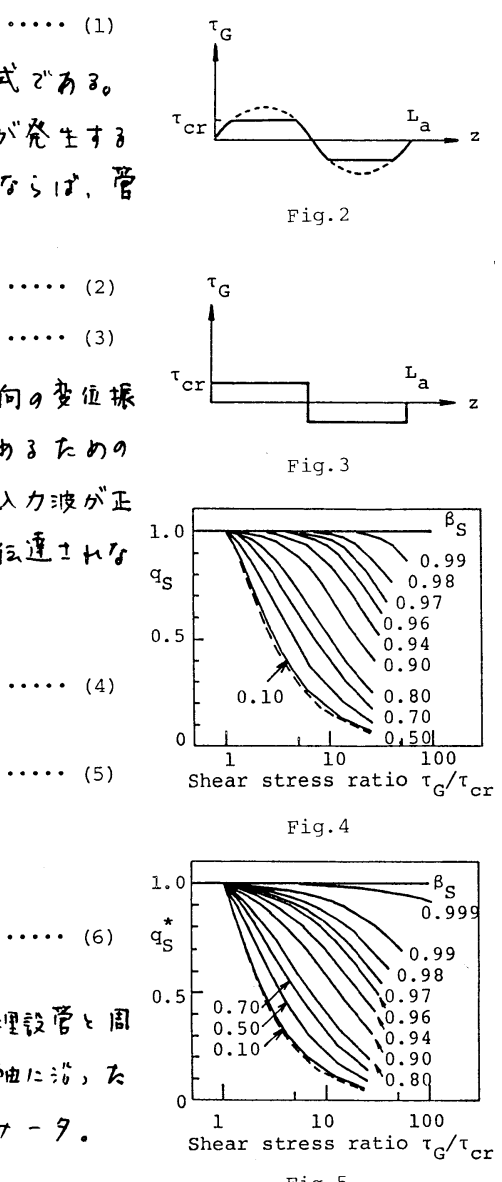

Fig. 2 


$$
\tau_{G} / \tau_{c r}=\left(1-q_{S}^{* \beta}\right)^{\prime S}
$$

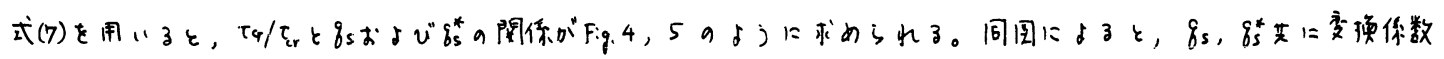

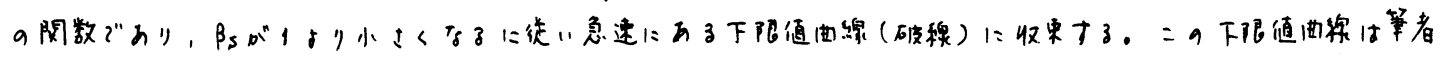

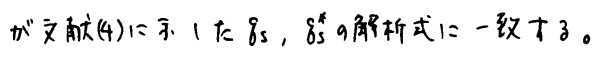

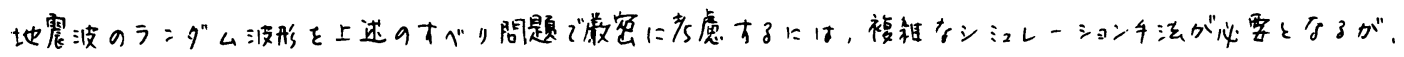

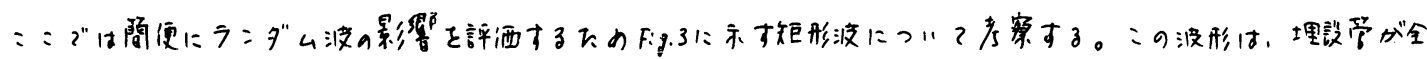

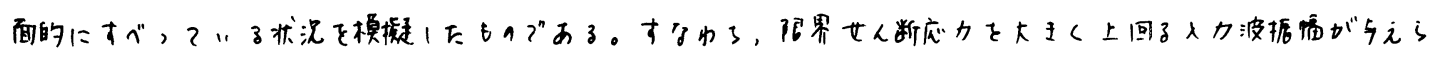

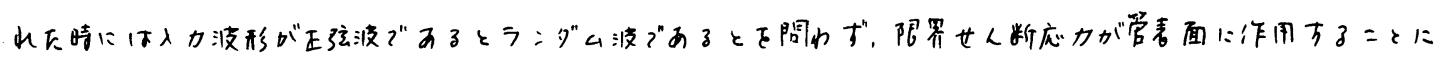

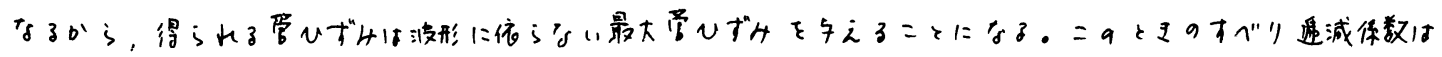
次式で求められ。

$$
\begin{aligned}
& q_{R}=\pi / 2 \cdot\left(1+\mathrm{K}^{2}\right) S \quad \ldots \cdots(8) \\
& q_{R}^{*}=\pi^{2} / 8 \cdot k^{2} S / B_{S} \quad \ldots \ldots \text { (9) }
\end{aligned}
$$

\section{3. 曲営部のUブ々解析}

Fig.6は曲管部に入カ波が入射角中で入射に大场含である。 入カ振幅の方向が蚰方向に一致する入波をRータイプ,

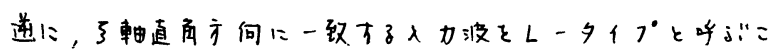

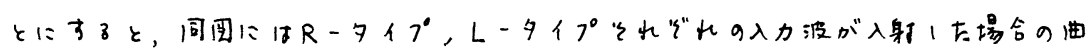

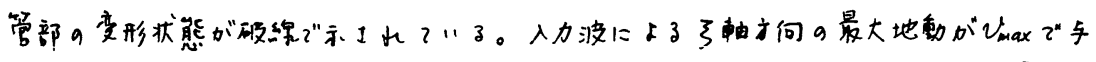

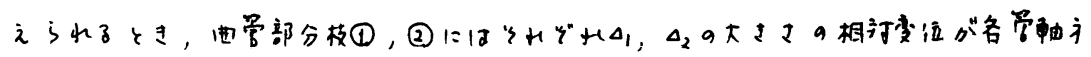

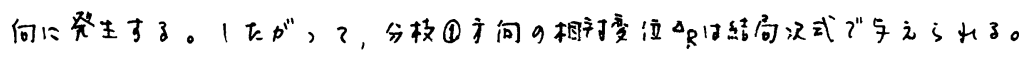

$$
\begin{aligned}
& \Delta_{R}(\phi)=\left|\Delta_{1}\right|-\left|\Delta_{2}\right| \cos \psi \quad-\psi \leqq \phi \leqq \pi-\psi \\
& ==2^{\prime \prime} \text {, } \\
& \underline{\mathrm{R}-\text { type }} \\
& \Delta_{1}=\left\{\cos \phi-\beta_{D}(\phi)\right\} v_{\max } \\
& \Delta_{2}=\left\{\cos (\pi-\psi-\phi)-\beta_{D}(\pi-\psi-\phi)\right\} v_{\max } \\
& \Delta_{1}=\left\{\sin \phi-\beta_{D}(\phi)\right\} v_{\max } \\
& \Delta_{2}=\left\{\sin (\pi-\psi-\phi)-B_{D}(\pi-\psi-\phi)\right\} v_{\max }
\end{aligned}
$$

ただし.

$$
\beta_{D}(\phi)=q_{S}^{*} \beta_{S}
$$

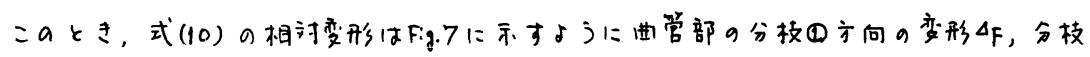

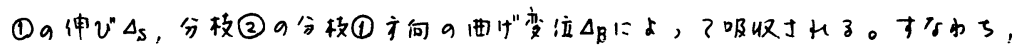

$$
\Delta_{R}(\phi)=\Delta_{F}+\Delta_{S}+\Delta_{B}
$$

水道・ガス用に一般に用いられる管径・管厚比のパイプで製作され下曲管では、 Fig.8に示するうに林端の曲げモーナ二トMに比例、て営が偏平し応力其中が登生する。

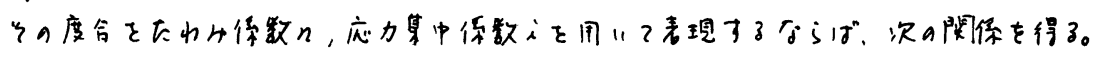

$$
\Delta \psi / \psi=n M R /(E I), \quad \sigma_{B}=i M / 2
$$

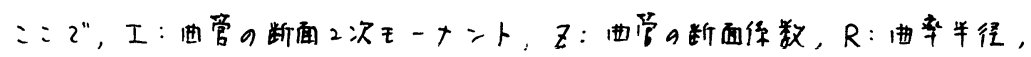
$\Delta \psi$ : 曲管角度变化

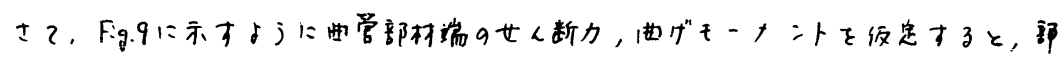
朴端での逨紿条件から次式飞得る。

$v_{1}(0)=0, v_{2}(0)=0, \theta_{1}(0)-\theta_{2}(0)=\theta_{B^{\prime}}, M_{1}=-M_{2}+R S_{1} \sin \psi+R S_{2}(1-\cos \psi)$

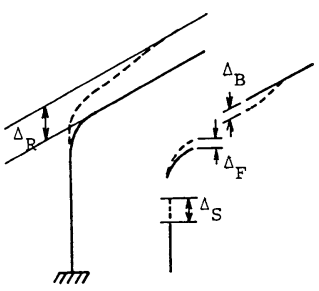

Fig. 7

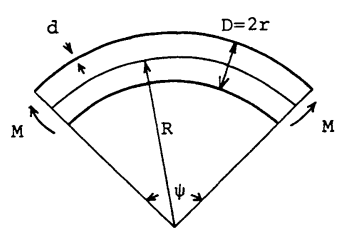

Fig. 8
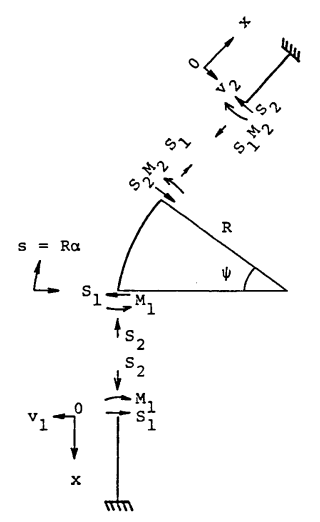


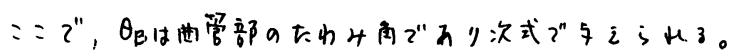

$$
\theta_{B}=\psi \frac{n R^{2}}{E I}\left[\frac{M_{2}}{R}+S_{1}\left(\frac{1-\cos \psi}{\psi}-\sin \psi\right)+S_{2}\left(\cos \psi-\frac{\sin \psi}{\psi}\right)\right]
$$

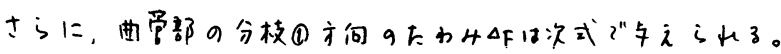

$$
\begin{aligned}
\Delta_{F}=\frac{n R^{3}}{2 E I} & {\left[\frac{2 M_{2}}{R}(\psi \cos \psi-\sin \psi)+2 S_{1}\left\{\cos \psi-\cos 2 \psi-\frac{\psi}{2} \sin 2 \psi+\left(1+\frac{I}{n R^{2} A}\right) \frac{\cos 2 \psi-1}{4}\right.\right.} \\
& +2 S_{2}\left\{\psi \cos ^{2} \psi-\sin 2 \psi+\left(1+\underset{n R^{2} A}{\longrightarrow} \frac{2 \psi+\sin 2 \psi}{4}\right\}\right]
\end{aligned}
$$

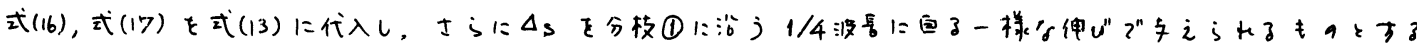

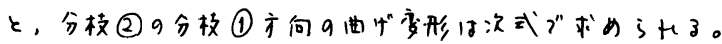

$$
\Delta_{B_{1}}=\frac{\Delta_{R}(\phi)}{1+\frac{L_{a_{1}} I \lambda^{3}}{2 A}\left|\frac{1+C_{2}+C_{1} \cos \psi}{\sin \psi}\right|+C_{3}}
$$

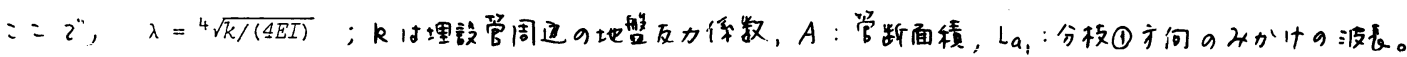

$C_{1}=\left(C_{22} a_{1}-C_{12} a_{2}\right) /\left(C_{11} C_{22}-C_{21} C_{12}\right), C_{2}=\left(C_{11} a_{2}-C_{21} a_{1}\right) /\left(C_{11} C_{22}-C_{21} C_{12}\right)$

$C_{3}=n R^{3}\left[\frac{2}{R}(\psi \cos \psi-\sin \psi) \lambda^{2} C_{2}-2 \lambda^{3} C_{1}\left\{\cos \psi-\cos 2 \psi-\frac{\psi}{2} \sin 2 \psi+\left(1+\frac{I}{n R^{2} A} \frac{\cos 2 \psi-1}{4}\right\}\right.\right.$

$\left.-2 \lambda^{3} \frac{1+C_{2}+C_{1} \cos \psi}{\sin \psi}\left\{\psi \cos ^{2} \psi-\sin 2 \psi+\left(1+\frac{I}{n R^{2} A}\right) \frac{2 \psi+\sin 2 \psi}{4}\right\}\right]$

$C_{11}=1+2 n R^{2} \lambda^{2}(1-2 \cos \psi+\psi \cos 2 \psi / \sin \psi), C_{12}=-1-2 n R^{2} \lambda^{2}\{1+\psi /(R \lambda)-\psi \cot \psi\}$

$C_{21}=1+R \lambda(\cot \psi-\cos 2 \psi / \sin \psi), \quad C_{22}=1+R \lambda(1-\cos \psi) / \sin \psi$

$a_{1}=-\sin \psi-2 n R^{2} \lambda^{2}(\psi \cos \psi-\sin \psi), \quad a_{2}=-\lambda R(1-\cos \psi)$

$\Delta_{B_{1}}$ そ用いると，女人断力，曲げ王一キ二トは次式で求のうれる。

$M_{1}=2 E I \lambda^{2} C_{1} \Delta_{B_{1}}, M_{2}=2 E I \lambda^{2} C_{2} \Delta_{B_{1}}, S_{1}=-2 E I \lambda^{3} C_{1} \Delta_{B_{1}}, \quad S_{2}=-2 E I \lambda^{3}\left(1+C_{2}+C_{1} \cos \psi\right) \Delta_{B_{1}} / \sin \psi \ldots \ldots$

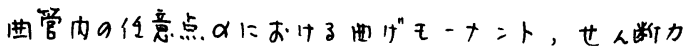

$M(\phi, \alpha)=M_{2}-S_{1} R(\sin \psi-\sin \alpha)-S_{2} R(\cos \alpha-\cos \psi)$

$S(\phi, \alpha)=S_{1} \sin \alpha-S_{2} \cos \alpha$

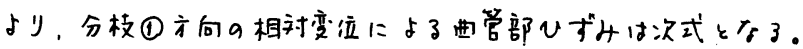

$$
\varepsilon_{B_{1}}(\phi, \alpha)=i \frac{D}{2 E I}|M(\phi, \alpha)|+\left|\frac{S(\phi, \alpha)}{A E}\right|
$$

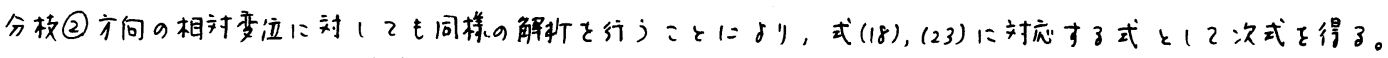

$$
\begin{gathered}
\Delta_{B_{2}}=\frac{\Delta_{R}(\psi-\phi)}{1+\frac{L_{a_{2}}^{*} I \lambda^{3}}{2 A}\left|\frac{1+C_{2}+C_{1} \cos \psi}{\sin (\psi-\phi)}\right|+C_{3}} \\
\varepsilon_{B_{2}}(\phi, \alpha)=i \frac{D}{2 E I}|M(\psi-\phi, \alpha)|+\left|\frac{S(\psi-\phi, \alpha)}{A E}\right|
\end{gathered}
$$

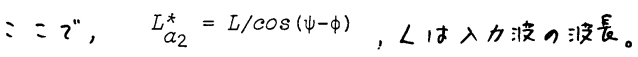

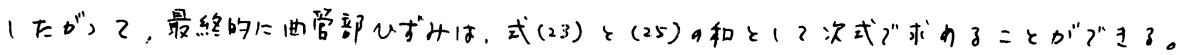

$$
\varepsilon_{B}(\phi, \alpha)=\varepsilon_{B_{1}}(\phi, \alpha)+\varepsilon_{B_{2}}(\phi, \alpha)
$$

乡はて，曲管に詨才子变换㐿数は

$$
\beta_{B}(\phi, \alpha)=\varepsilon_{B}(\phi, \alpha) / \varepsilon_{G}
$$

$と \tau_{子} る$ 


\section{4. 数值言草例}

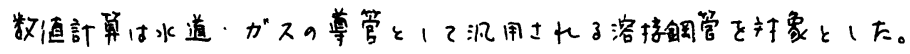
使用铜管の諸㭙性を Talde 1 に示才。

埋設地盤はTable2に示す了種颣の地盤飞打象とし，埋没管表面での限界

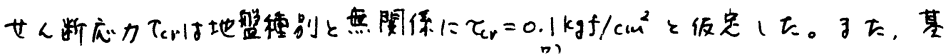

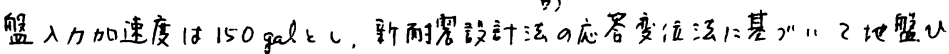
ずНと章定した。

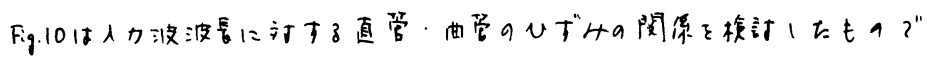

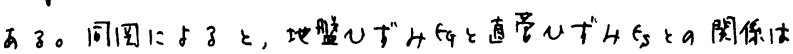

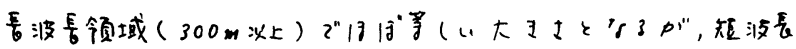

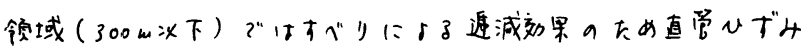

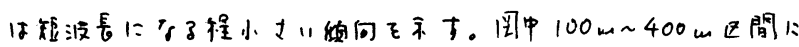

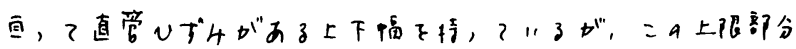

\begin{tabular}{|c|c|c|c|c|c|}
\hline \multirow{2}{*}{ 地 馧 諸 } & \multicolumn{2}{|l|}{ 元 } & \multicolumn{3}{|c|}{ 表屈地盤 } \\
\hline & & & & & \\
\hline 単位体積重量 & $\rho_{G}$ & $\mathrm{kgf} / \mathrm{cm}^{3}$ & $1.8 \times 10^{-3}$ & $1.8 \times 10^{-3}$ & $1.8 \times 10^{-3}$ \\
\hline 卓越周期 & $\mathrm{T}$ & $\mathrm{sec}$ & 1.0 & 0.5 & 0.2 \\
\hline 限界せ九断応力 & $\tau_{\mathrm{G}}$ & $\mathrm{kgf} / \mathrm{cm}^{2}$ & 0.1 & 0.1 & 0.1 \\
\hline 地盤反力绿数 & $\mathrm{k}$ & $\mathrm{kgf} / \mathrm{cm}^{2}$ & $1.5 \pi \mathrm{D}$ & $1.5 \pi$ & $1.5 \pi \mathrm{D}$ \\
\hline
\end{tabular}

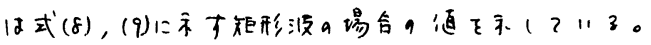
:の国にはると，直管ひボムに与之るランダム波形

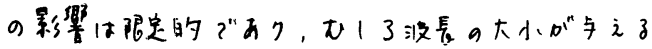

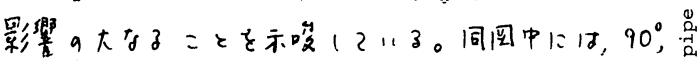

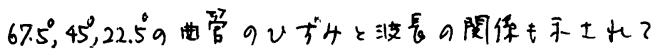

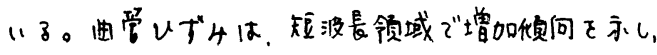

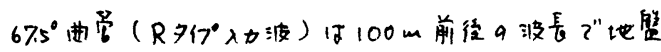

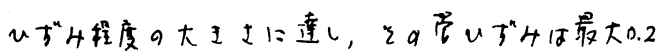
$\%$ に逢いている。
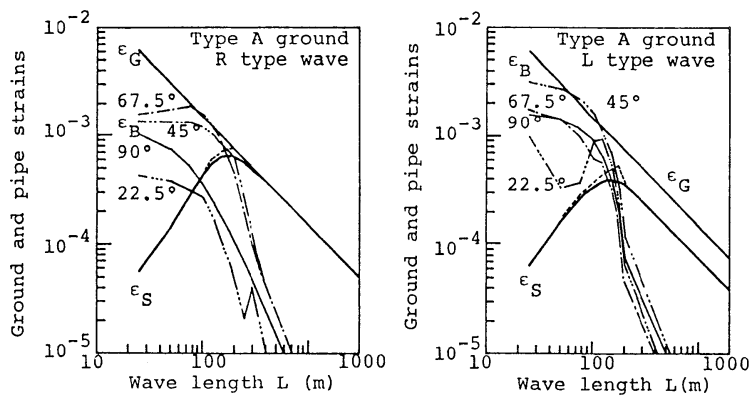

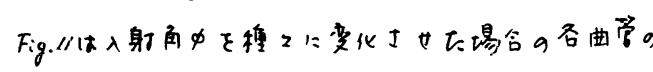

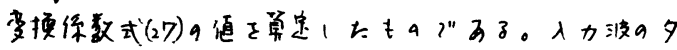

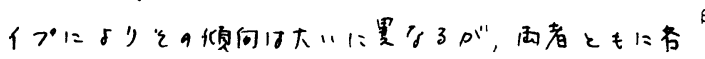

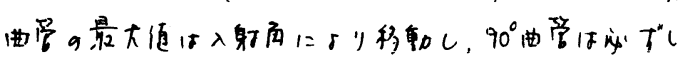

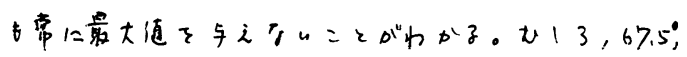

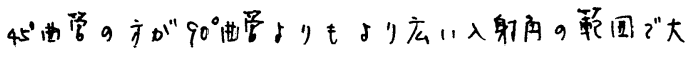

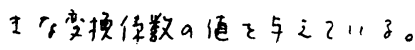

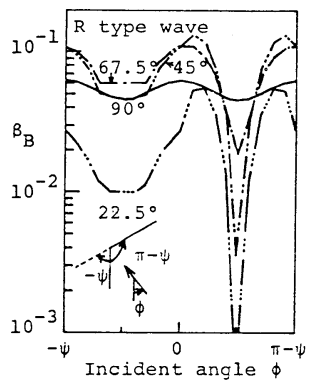
Fig. 10

\begin{tabular}{lll|l}
\hline \multicolumn{1}{c|}{ 諸 } & 元 & & \\
\hline 管径 & & $\mathrm{D}_{\mathrm{mm}}$ & 600 \\
管厚 & $\mathrm{d}_{\mathrm{mm}}$ & 6 \\
曲率半径 & $\mathrm{R}$ & $1.5 \mathrm{x}$ \\
パイプファクター & $\mathrm{h}$ & 0.06 \\
応力集中係数 & $\mathrm{i}$ & 9.38 \\
たわみ你数 & $\mathrm{n}$ & 27.5 \\
\hline
\end{tabular}

5. 万とがす

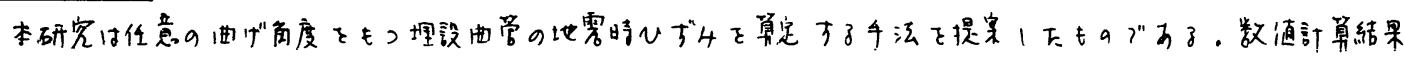

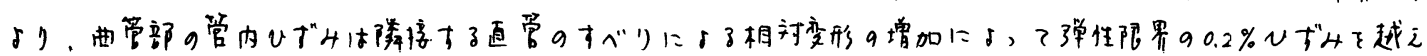

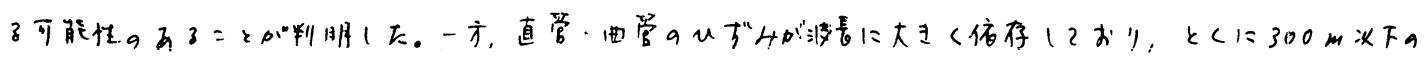

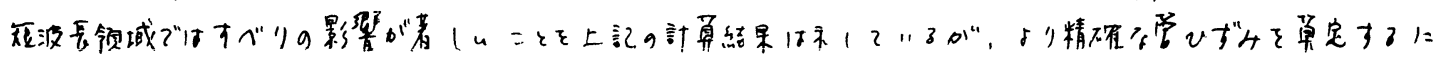

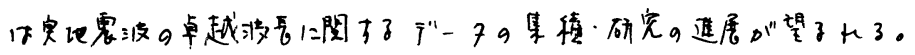

\section{参考文献}

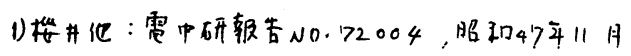

2) SHAH, CHU: AS CE, PO1, pp. 53-62, 1974

3) SHiwozUkA, KoIKE : ASMIE Publication, pp.3!-48, 1979

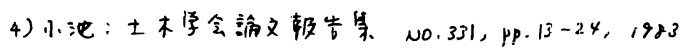

5) NYMAN : US-JApan Workshop on Buried Pipplimes, Toukuba ff4

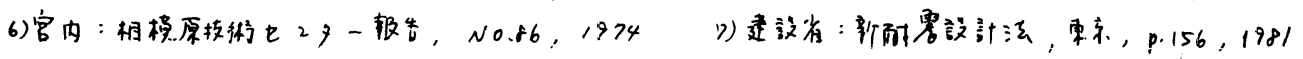

